\title{
Studying User Experience of a Hybrid Location Sensing System
}

\author{
Yun Huang \\ Syracuse University \\ Syracuse, NY, USA \\ yhuang@syr.edu

\section{Qunfang Wu} \\ Syracuse University \\ Syracuse, NY, USA \\ qwu114@syr.edu
}

\author{
Yisi Sang \\ Syracuse University \\ Syracuse, NY, USA \\ yisang@syr.edu \\ Yaxing Yao \\ Syracuse University \\ Syracuse, NY, USA \\ yyao08@syr.edu
}

\section{ABSTRACT}

The $\mathrm{HCl}$ community has extensively studied location-based systems that applied various location sensing technologies. However, there is a lack of user experience (UX) studies of systems where hybrid location sensing approaches are provided. In this work, we designed, implemented and studied a hybrid location sharing system that offers automatic location sensing through Bluetooth Low Energy (BLE) beacons and participatory location sharing using GPS. Our findings provide design implications to future location-based systems that apply such a hybrid location sensing design.

\section{CCS CONCEPTS}

- Human-centered computing $\rightarrow$ User studies.

Permission to make digital or hard copies of part or all of this work for personal or classroom use is granted without fee provided that copies are not made or distributed for profit or commercial advantage and that copies bear this notice and the full citation on the first page. Copyrights for third-party components of this work must be honored. For all other uses, contact the owner/author(s)

CHI'19 Extended Abstracts, May 4-9, 2019, Glasgow, Scotland Uk

(c) 2019 Copyright held by the owner/author(s).

ACM ISBN 978-1-4503-5971-9/19/05.

https://doi.org/10.1145/3290607.3312793 


\section{KEYWORDS}

Location Sensing; Participatory Sensing; Opportunistic Sensing; Bluetooth Low Energy Beacon

\section{INTRODUCTION}

The $\mathrm{HCl}$ community has extensively studied location-based community systems that applied various location sensing technologies and these technologies can be classified into two major categories, i.e., absolute and proximity-based location sensing [10]. For example, GPS is one of the absolute technologies that allow users to share their location with latitude and longitude at any given moment. On the other hand, Bluetooth Low Energy (BLE) beacon is a leading proximity-based location sensing technology that requires phones' Bluetooth to be on, then automatically senses whether a user is close to a location where a beacon is installed (e.g., [5]).

Prior systems mostly applied only one sensing technology, and very few location-based systems applied hybrid sensing solutions [8]. Those that applied hybrid sensing approaches either did not study user behaviors (e.g., [13]) or could not support automatic proximity-based location sensing (e.g., [7]). Additionally, current studies of BLE beacons focus on addressing technology issues and proposing beacon applications in different domains (e.g., promoting in-store sales [15], managing energy consumption [3], and organizing crowds [6]), instead of understanding how users adopt them.

In this work, we present our design, implementation and study of a hybrid location sharing system that offers both automatic location sensing through BLE beacons and manual location sharing using GPS. More specifically, the system is applied by college students to take their class attendance. This application is motivated by the fact that BLE beacons have been proposed to automate students' class attendance-taking in classrooms, but none of the prior work $[1,4,14]$ reported user experience (UX) on how students really used BLE beacon-based solutions for attendance-taking in classrooms. Our preliminary work showed that only using BLE beacons failed with low app adoption [9], as BLE beacon-based location sensing requires users to always share their location with the app even the app is not in use and some people have privacy concerns of doing so. Our follow-up study showed that a hybrid location sensing solution with two sensing approaches increased the overall app adoption; however, user experience was not reported in our paper [9]. In this work, we conducted a 2-month field trial study with 101 student participants and studied how they applied these two sensing approaches to share their class attendance with their instructors. The findings have broader implications to designing hybrid location sensing systems.

\section{System Design}

Figure 1 illustrates the system model with screenshots of a mobile app and a web interface. When a student first launches the mobile app, it sends a request to the attendance server to look up the 


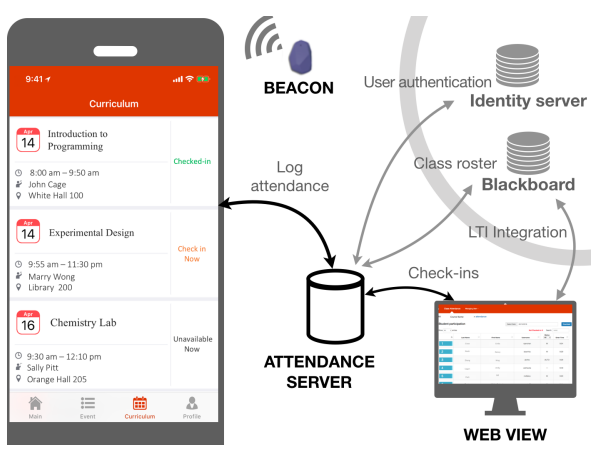

Figure 1: An illustration of the system model. The system provides a mobile app that students can use to check in to classes and a web interface where instructors can review and edit attendance records. In this work, we focused on examining how student participants used the mobile app to check in to classes (class content displayed on the app screenshot was revised for anonymity). Privacy concerns are carefully addressed in the design. For example, each student' records are only accessible to the instructor and the app does not sense participants' location beyond the classroom or outside the lecture time. student's class information. The server returns course title, time, instructor, and classroom location The app screenshot shows the interface where students can find their classes and check-in statuses. Before attendance is taken, if the present time is within the check-in window, then the status is in orange, suggesting the student is not checked in yet; otherwise, the status is grey "unavailable." Once attendance is taken, the status will be changed to green, meaning the student has successfully checked in. To automate class attendance-taking, BLE beacons are installed in the classrooms and the app keeps the beacons' identifiers for the classrooms. BLE beacons are small devices that broadcast Bluetooth signals. When the phone's Bluetooth is on and location sharing is allowed, the phone will sense the beacons' signals. Once the app detects the beacon's signal around the lecture time, then it suggests that the student enters the classroom and the app logs the student's attendance to the server Instructors may have different rules on when students can check in to a class. Our system allows students to check in 15 minutes before the class has started and 15 minutes before the class is over.

\section{METHOD}

When recruiting student participants, we needed to make sure students knew that the research was not related to their courses and whether or not to participate in the study would not impact their grades. These would only be confirmed by their instructors. Thus, we first sent out messages to our school's listserv to seek instructors who would be willing to pass the recruitment script on to their students. We targeted classes where student attendance was already included as part of their final grades. In this case, only their attendance mattered to the grades; their decision whether to use the app (and participate in the study) and whether to participate in the follow-up interviews would not impact their grades. Using the app in each class was voluntary during the field study period. Throughout the study, the system was instrumented to reliably log users' behaviors (e.g., checking in to a class using a particular method) with corresponding timestamps.

After each field trial, participants were eligible to be invited to a one-hour semi-structured interview. The interviews were designed to understand participants' app adoption and usage and to collect their feedback about the system design. We tried to diversify the interviewees based on their app usage (auto and manual check-ins), phone platform (iOS/Android), and class time (morning/afternoon) Interviewees' app usage and phone platform were provided by system log analysis. Each participant was paid $\$ 5$ for finishing one interview. All the interviews were audio recorded with interviewees' consent and all the audio interviews were transcribed. We conducted a thematic analysis [2] that was commonly used to analyze qualitative data. This project is approved by our university's IRB.

Eventually, 48 undergrads ( 13 female, 35 male) and 53 graduate students ( 29 female, 24 male) from three different courses participated in the field trial. Ages of the student participants varied between 19 and 25 (averaged at 20). In each class, the instructors used the web interface to review/update students' attendance, so that those who did not participate in the studies and those participants who 
Sample case 2: U19 (female) began with manual check-ins to learn how the app worked. Soon she switched to auto checkins and kept using it thereafter: "I manually check in only for the first two weeks. Now I don't manually check in, because I know it [auto checkin] worked and just love it. I'm getting used to the system... Now, I prefer Auto check-in. It's easy because of the time management. If I am still on my phone [manually] checking in, I can't concentrate [on the lecture].”

Sample case 1: U15 (male) started with manual check-ins to build trust with the system. Once he figured that auto checkin worked and its benefits, he became comfortable with auto check-ins and kept using the auto sensing approach: "For the first several times, I just manual checked in because I didn't trust Bluetooth. But after certain times, I forgot to check in and when I opened the app the app already checked (me) in automatically, so I trusted it and kept using the auto check-in method." did not check in through the app could still have their attendance confirmed by the instructors. A total of 20 participants (nine male, 11 female) were interviewed.

\section{FINDINGS}

Overall, participants used beacon-based auto check-ins more frequently than manually taking class attendance by sharing their GPS location. Privacy is always a major issue of location-based sensing systems and we discussed privacy concerns of using BLE beacons in our prior work [9, 16]. In this paper, we mainly discuss new findings where participants' feedback revealed that the manual approach was helpful for the adoption of the automatic approach. Our results also suggested app usage varied over different times of day.

Usage of the Two Sensing Approaches. The undergraduate participants had 14 lectures throughout the field trial, with 326 auto check-ins and 115 manual check-ins in total. On average, each undergraduate participant checked in to $93 \%(S D=0.11)$ of his/her classes using the app. The graduate participants had 7 lectures in total. Still, there were more auto check-ins (195) than manual check-ins (147). On average, each graduate participant checked in to $95 \%(\mathrm{SD}=0.10)$ of his/her classes using the app.

Switching Between the Two Sensing Approaches. Given the two sensing approaches, more than half of the participants switched between manual and automatic check-ins, i.e., 64 participants (63\%) switched between the two sensing approaches. About 30 participants $(30 \%)$ always checked in automatically; only seven participants (7\%) kept checking in manually all the time.

An important finding was that there were participants (e.g., U15, U19) who started with manual check-ins and then switched to auto check-ins in later weeks. Interviews of the participants explained they transitioned for different reasons. For example, as the sample cases present in the side bar, some used manual check-ins to learn how the system works, and some took the approach to build trust.

On the other hand, there were cases where participants (e.g., U10, U53) switched from auto to manual check-ins. For example, U10 (female) chose the check-in approach based on her availability. Her first check-in was made automatically because she was late for that class, but she went to classes earlier thereafter and then she manually checked in to them all: "Because I usually arrive early, so I prefer to check in manually, because I have time. When I arrived late, I didn't want to use cell phone, then Bluetooth [auto check-in] can help.”

Manual Check-ins Differed Between Varied Use Times. Some participants shared why they preferred manual check-ins instead of auto check-ins in the afternoon. It was because their phones were about to run out of battery and they thought the automatic approach drained the battery more quickly than the manual approach. For example, U53 (female) took the manual approach as she concerned that her afternoon class lasted three hours: "Because my battery will run out easier." We then took the system 
Table 1: GPS manual check-ins from participants who took morning and afternoon classes.

Mean SD

\begin{tabular}{rrr} 
& Mean & SD \\
\hline Morning & 2.55 & 1.88 \\
Afternoon & 3.84 & 1.95 \\
\hline
\end{tabular}

logs of the participants' attendance and compared the use of auto/manual check-ins between the morning and afternoon classes. The result of Kruskal-Wallis rank sum test showed that there was a significant difference between the number of manual check-ins in the morning and afternoon classes $(H=4.70, p<0.05)$, as shown in Table 1.

\section{DISCUSSION}

The automatic, also called opportunistic, sensing approach does not require users' explicit participation, which is typically used when it is difficult to keep constant user participation. On the other hand, the manual, also called participatory, approach requires users' explicit participation, but gives users more flexibility by allowing them to decide when to share data and how privacy mechanisms are to be set up each time $[8,10]$.

Our work revealed a new promoting interaction between the two sensing approaches-that providing a participatory sensing approach had a positive effect on the adoption of opportunistic sensing. More specifically, as shared by our participants, after taking the participatory sensing approach (checking in to classes manually), some participants had a better understanding and more trust with the hybrid sensing system, which in turn promoted participants' use of the opportunistic sensing approach enabled by BLE beacons. This new relationship between the two sensing approaches has design implications in more diverse contexts. For example, when collecting noise spots [12] and real-time traffic conditions [11], both opportunistic (different sensors on smart phones can be used to automatically collect relevant information) and participatory (users explicitly share their observed data) approaches can be applied together. Our findings suggest that it may be necessary to integrate a participatory approach to help users better understand and adopt the opportunistic approach.

Our findings also suggested that participants' concerns of using the BLE beacon-based location sensing approach varied between morning and afternoon, which resulted in different use of the two location sensing approaches. Therefore, the time-of-use factor may need to be considered when analyzing user behavior data of hybrid location sensing systems.

Additionally, we observed that the iOS participants used auto sensing more frequently than android participants. In our future work, we plan to further investigate more factors, including phone platforms and attendance requirement on user experience with the hybrid sensing design.

\section{ACKNOWLEDGEMENTS}

This research was supported by a Google Faculty Research Award. This material is also based upon work supported by the National Science Foundation under Grant No. \#1464312. Any opinions, findings, and conclusions or recommendations expressed in this material are those of the author(s) and do not necessarily reflect the views of the National Science Foundation. 


\section{REFERENCES}

[1] Mi-Young Bae and Dae-Jea Cho. 2015. Design and implementation of automatic attendance check system using BLE beacon. International Journal of Multimedia and Ubiquitous Engineering 10, 10 (2015), 177-186.

[2] Richard E Boyatzis. 1998. Transforming qualitative information: Thematic analysis and code development. Sage.

[3] Andrea Corna, L Fontana, AA Nacci, and Donatella Sciuto. 2015. Occupancy detection via iBeacon on Android devices for smart building management. In Proceedings of the 2015 Design, Automation \& Test in Europe Conference \& Exhibition. EDA Consortium, 629-632.

[4] Dwight Deugo. 2016. Using Beacons for Attendance Tracking. In Proceedings of the International Conference on Frontiers in Education: Computer Science and Computer Engineering (FECS). The Steering Committee of The World Congress in Computer Science, Computer Engineering and Applied Computing (WorldComp), 155.

[5] Estimote. 2018. Estimote Bluetooth Low Energy Beacon. https://estimote.com/

[6] Emanuele Frontoni, Adriano Mancini, Roberto Pierdicca, Mirco Sturari, and Primo Zingaretti. 2016. Analysing human movements at mass events: A novel mobile-based management system based on active beacons and AVM. In Control and Automation (MED), 2016 24th Mediterranean Conference on. IEEE, 605-610.

[7] Bin Guo, Zhiwen Yu, Xingshe Zhou, and Daqing Zhang. 2014. From participatory sensing to mobile crowd sensing. In Pervasive Computing and Communications Workshops (PERCOM Workshops), 2014 IEEE International Conference on. IEEE, 593-598.

[8] Yun Huang, Alain Shema, and Huichuan Xia. 2017. A proposed genome of mobile and situated crowdsourcing and its design implications for encouraging contributions. International Journal of Human-Computer Studies 102 (2017), 69-80.

[9] Yun Huang, Qunfang Wu, and Yaxing Yao. 2018. Bluetooth Low Energy (BLE) Beacons Alone Didn't Work!. In Proceedings of the 2018 ACM International Joint Conference and 2018 International Symposium on Pervasive and Ubiquitous Computing and Wearable Computers, UbiComp/ISWC 2018 Adjunct, Singapore, October 08-12, 2018. 62-65. https://doi.org/10.1145/ 3267305.3267634

[10] Quentin Jones, Sukeshini A Grandhi, Loren Terveen, and Steve Whittaker. 2004. People-to-people-to-geographical-places: the P3 framework for location-based community systems. Computer Supported Cooperative Work (CSCW) 13, 3-4 (2004) 249-282.

[11] Emmanouil Koukoumidis, Li-Shiuan Peh, and Margaret Rose Martonosi. 2011. SignalGuru: leveraging mobile phones for collaborative traffic signal schedule advisory. In Proceedings of the 9th international conference on Mobile systems, applications, and services. ACM, 127-140.

[12] Nicolas Maisonneuve, Matthias Stevens, Maria E Niessen, and Luc Steels. 2009. NoiseTube: Measuring and mapping noise pollution with mobile phones. In Information Technologies in Environmental Engineering. Springer, 215-228.

[13] Emiliano Miluzzo, Nicholas D Lane, Kristóf Fodor, Ronald Peterson, Hong Lu, Mirco Musolesi, Shane B Eisenman, Xiao Zheng, and Andrew T Campbell. 2008. Sensing meets mobile social networks: the design, implementation and evaluation of the cenceme application. In Proceedings of the 6th ACM conference on Embedded network sensor systems. ACM, 337-350.

[14] Shota Noguchi, Michitoshi Niibori, Erjing Zhou, and Masaru Kamada. 2015. Student attendance management system with bluetooth low energy beacon and android devices. In Network-Based Information Systems (NBiS), 2015 18th International Conference on. IEEE, 710-713.

15] Mirco Sturari, Daniele Liciotti, Roberto Pierdicca, Emanuele Frontoni, Adriano Mancini, Marco Contigiani, and Primo Zingaretti. 2016. Robust and affordable retail customer profiling by vision and radio beacon sensor fusion. Pattern Recognition Letters 81 (2016), 30-40.

[16] Yaxing Yao, Yun Huang, and Yang Wang. 2019. Unpacking People's Understandings of Bluetooth Beacon Systems - A Location-Based loT Technology. In System Sciences (HICSS), 2019 52nd Hawaii International Conference on. IEEE. 